\title{
A quantitative model to define the priority of recovery of road network elements following a relevant event
}

\author{
Fabio Borghetti \\ Mobility and Transport Laboratory, Politecnico di Milano, Italy.E-mail: fabio.borghetti@polimi.it \\ Marco Ponti \\ Mobility and Transport Laboratory, Politecnico di Milano, Italy.E-mail:marco1.ponti@polimi.it
}

Giovanna Marchionni

Mobility and Transport Laboratory, Politecnico di Milano, Italy.E-mail: giovanna.marchionni@polimi.it

Luca Studer

Mobility and Transport Laboratory, Politecnico di Milano, Italy.E-mail: luca.studer@polimi.it

Paolo Gandini

Mobility and Transport Laboratory, Politecnico di Milano, Italy.E-mail: paolo.gandini@polimi.it

Roberto Maja

Mobility and Transport Laboratory, Politecnico di Milano, Italy.E-mail: roberto.maja@polimi.it

\begin{abstract}
The work proposes a quantitative model to determine the transport relevance of the different sections of a road network. In this way it is possible to define priorities for single network elements' restore following relevant events such as earthquakes. The model was developed within a project with the Italian Civil Protection Department following the earthquakes occurred in central Italy in 2016. The planning and programming of the interventions arises from the need to restore in the shortest time the ordinary people mobility within the territory; in the meantime it is also necessary to guarantee the operational needs of the Civil Protection, such as the transport and construction of temporary housing for the population. The proposed model considers the development of 5 quantitative indicators to characterize the transport importance of the network links deciding the priority of the interventions. These indicators take into account, for example, the link flow, the number of paths over a link and the Origin/Destination pairs satisfied. In this way it is possible to assess the impact that the restore of a network link could have on one or more routes and generally on the whole road network. The model can be considered as a Decision Support System that can be used to plan and schedule the restore of a road network according to specific needs.
\end{abstract}

Keywords: Transport vulnerability, civil protection, emergency management, road network, disaster recovery, decision support system, traffic assignment, traffic flows, transport demand, transport supply.

\section{Introduction}

When a major event occurs over a larger or smaller area, the transport system plays a key role in emergency management. The rescue system, in fact, can use different modes of transport in order to achieve two main objectives: to bring relief to the affected areas in the least possible time and to evacuate the population from the affected areas. In the post-emergency phase, the priority is to recover the operation of the transport network, which may have been partially or completely damaged by the event itself. This activity is aimed, on the one hand, to ensure the main movements of the vehicles involved in the activities of reconstruction and safety of the territory and the population (demolition, rubble removal, construction of temporary housing modules, etc.), on the other hand, to restore the possibility of moving people and goods that affect the territory. The latter aspects can have an important impact on the socio-economic system related to a region or country; it is therefore important to bring the functionality of the network to an acceptable level as soon as possible. Considering the interdependencies, the functioning of infrastructure systems (e.g. transportation, power, water and telecommunications) depends on each other, thus interruptions in one system can spread to other systems and cause cascading failures, $\mathrm{Ni}$ et al. (2019). In a limited resources context, it is necessary to proper undertake a planning and schedule of recovery activities, in order to give priority to the interventions on those elements of 
the network characterized by significant importance compared to the overall functioning of the network itself. Having said that, it is necessary to define quantitative criteria allowing an estimate of the expected (positive) impacts on the entire network to follow the restoration of a single damaged element that helps to define the level of priority of the interventions themselves.

\section{Background}

In the technical-scientific literature several authors have addressed the issue of vulnerability and resilience of transport systems following relevant events. Today it is very difficult to give a unique definition of terms such as resilience, vulnerability and other components. In particular, the study of the vulnerability of a transport network allows to evaluate the serviceability that describes the possibility to use that link/route/road network during a given period, Berdica (2002) and Jenelius et al. (2006). Regarding the risk definition, vulnerability can be considered an element together with the probability of occurrence and the consequences of a dangerous event, Coburn et al. (1994) and Linkov et al. (2018). A network element can be considered vulnerable if its functionality's leads to a significant reduction in the accessibility of the network or particular elements, Taylor et al. (2006). Zio et al. (2008), proposed to study the robustness of network systems by analyzing the structure of interconnection of their components. They introduced a global and local reliability using efficient indicators for evaluating the robustness and vulnerability to faults of the network defining the reliability distances accounting for the probabilities of failure of the links interconnecting the nodes of the network. Others, like Ganin et al. (2016), propose a resilience evaluation method based on critical functionality, a performance function of time set by the stakeholders. With reference to the definition of resilience, the components related to response and recover have been deeply studied. The proposed model has been developed according to the above-mentioned analyses. In fact, as shown in Fig. 1, the response and recovery phases of a transport system can present different trends depending on time, Zhang et al. (2017). The time depends on several factors such as, for example, accessibility of the territory, availability of specialized resources and equipment, planning of interventions, etc., Borghetti and Malavasi (2016a), Borghetti and Malavasi (2016b). With reference to Fig. 1, the proposed model aims to identify the curve that will allow the recovery of the infrastructure in the shortest possible time according to the available resources, Borghetti et al. (2017). Ni et al. (2019) examine the routing problem of local supply chains delivering critical commercial services within an area under disrupted transportation systems.

Fig. 1. Different trajectories (trends) of the transport system

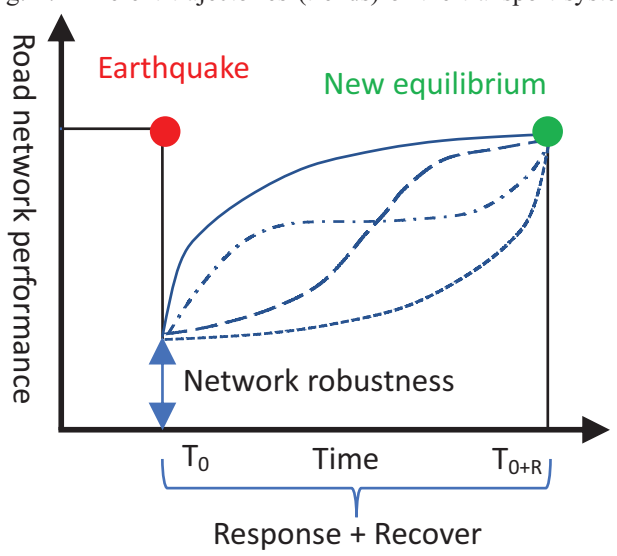

recovery as a function of time. Adapted from Carlson et al. (2012), Zhang et al. (2017) and Borghetti et al. (in press).

\section{Research goal and work structure}

The main goal of the work is to implement a Decision Support System - DSS capable of providing guidance to rank the interventions to be carried out for the recovery of a road network following relevant events. The tool, developed in the framework of an agreement with the Italian Civil Protection Department, allows to support different subjects, including road infrastructure managers in identifying the high priority recovery operations. In this way it is possible to allocate economic resources in an optimal and targeted way also according to time. In the event of an earthquake, for example, the number of transport infrastructures involved may be high. Recovery activities cannot be carried out simultaneously, but must be planned and programmed over time, according to the economic and operational resources available by defining certain priority criteria. The proposed model foresees two calculation sub-models: the first one concerns the development of indicators needed to guarantee the ordinary mobility of people and goods; the second one considers the specific needs of Civil Protection (provision of temporary housing modules, removal of rubble, securing the territory, etc.). The combination of the two submodels allows to obtain a global indicator of transport-wise importance for the different sections of the road network: in this way, when defining the priority classes of interventions, it is possible to consider both needs at the same time. The first sub-model is illustrated in this work and in particular the five indicators developed are described analytically. Fig. 2 shows the framework and the research phases described in the following paragraphs. 


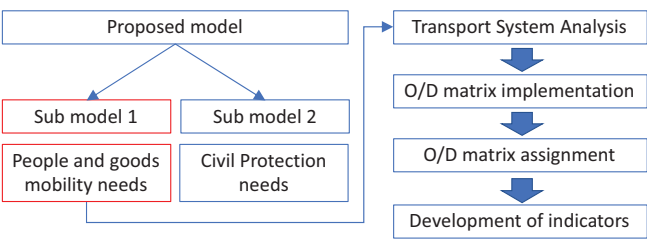

Fig. 2. Framework and structure of the proposed model.

\section{Analysis of the transport system and mobility demand}

Following the earthquakes that occurred in Italy in 2016, the National Civil Protection Department coordinated road restoration work. In four Italian regions, Lazio, Marche, Umbria and Abruzzo, earthquakes have caused significant damage, in some cases it resulted in a road no longer viable. The territorial area affected by the seismic events, which is the focus of this study (see Fig. 3), includes 131 municipalities and is characterized by an overall development of the road network of about $15,300 \mathrm{~km}$.

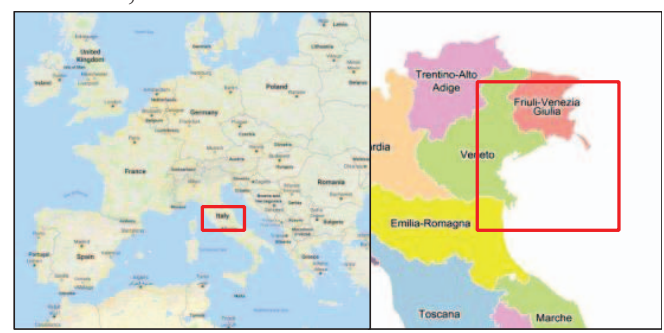

Fig. 3. Territorial representation of the study area where the proposed model was applied.

The first step is to analyze the road transport system and the territorial area. The components of supply and transport demand have been defined and an analytical representation of their components has been used. In this phase GIS Geographic Information System tools and traffic simulation models were used. The transport supply was represented by means of a georeferenced graph of the transport network, where each road section is represented by a link; each link is associated with different information such as length, travel speed, number of lanes, etc.

In order to analyze the transport demand, the territory affected by the earthquake has been divided into areas generally coinciding with the municipalities or parts of them. Each zone represents an origin and/or destination of the journeys affecting the study area; a centroid, i.e. a dummy point representing the position of origin and destination of the journeys, has been associated to each of them. The estimation of the number of travels for each pair of origin/destination $\mathrm{O} / \mathrm{D}$ zones was made using a gravity model, a classical model widely used to calculate commuting patterns by many authors, such as Ganin et al. (2017). The estimated demand flow for the different O/D relations has not been calibrated and therefore does not represent a significant value in absolute terms, but it is significant in relative terms; for the aims of this work it is necessary to be able to compare the relative flows for the different O/D pairs. In this way it is possible to determine the territorial relationships that have a higher number of journeys than the other relationships. On the basis of these relationships it is possible to define the mobility needs of crossing, distribution and access for the territory affected by a relevant event. The estimated number of trips has been represented by an O/D matrix, where in each cell the number (flow) of journeys $f^{\text {fd }}$ generated by the $o$ origin zone, corresponding to the element row, and directed to the $d$ destination zone, corresponding to the element column, is indicated. By way of example, Fig.4 shows a study area divided into 4 origin/destination zones, represented by the corresponding centroids.

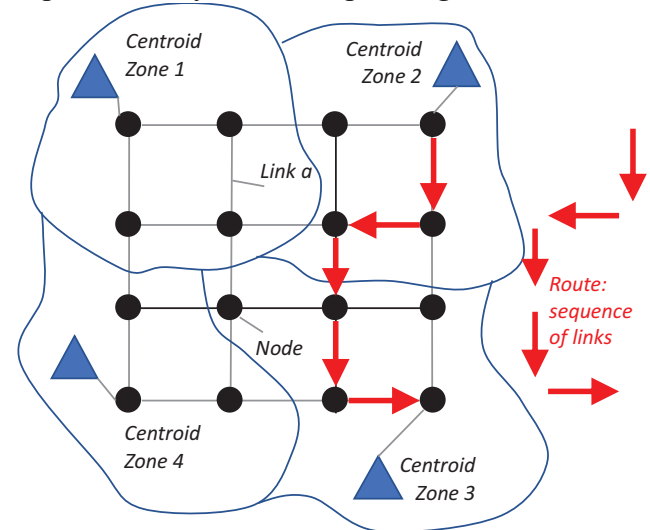

Fig. 4. Example of a territory being divided into 4 zones. In each zone is placed a centroid (triangle) representing the origin and destination of the journeys. The transport network is represented by links and nodes.

\section{O/D matrix assignment}

After analyzing and defining the supply and demand transport components, the next step is to assign the transport demand to the road graph. This step has been carried out using a deterministic All or Nothing algorithm, which assumes that all users moving between a given $\mathrm{O} / \mathrm{D}$ pair choose the route characterized by the lowest overall cost. In the proposed model, the generalized cost is represented by the travel time of each link of the network; road congestion, which could increase the travel time of a route, has been neglected as it is considered not relevant for the purpose of this research. At the end of the assignment procedure, it is possible to know: 
- $\quad t^{o d}[\mathrm{~min}]$ : the travel time of the routes connecting each $\mathrm{O} / \mathrm{D}$ pair;

- the sequence of the links crossed by the routes;

- $f_{a}^{o d}$ : traffic flow on each generic link $a$ generated by a given route $O / D$;

- $f_{a}$ : total flow on a generic link $a$, generated by the sum of all route flows passing through the link itself;

- $\quad N_{P a}$ : total number of routes crossing a generic link $a$.

\section{Transport importance indicators for road links}

The most important phase of the work is to develop a quantitative indicators to define the transport-wise importance of the different links (road sections) that make up the road network of the study area. The links characterized by the greatest transport importance are those where the presence of any critical issues and/or interruptions will have to be solved in a priority way compared to the other links. As already mentioned, in this work the five indicators developed are described in an analytical way, like shown below. Each of the 5 indicators is adimensional and can vary between 1 and 3 ; value 1 represents a high importance, value 2 a medium importance while 3 identifies a situation of low importance where the priority of recovery is not elevated. In a real application, the choice of the indicator to be used may depend on the purposes considered as priorities by the institutional and administrative subjects involved in the decision-making process, as well as on the availability of means and resources to carry out a high number of interventions in a short time.

\subsection{Origin-destination relationships - IOD}

The Origin-Destination relationships indicator $I_{O D}$ allows to associate a high priority of intervention to the paths that connect the areas of the territory characterized by a high demand for mobility, avoiding discontinuity and interruptions. The indicator guarantees that for $O / D$ relations characterized by high trips there is at least one route at minimum cost whose intervention priority is defined as high (value 1). It follows that all the links making up the route are characterized by the same intervention priority (high). Starting from the $O / D$ matrix, implemented with the gravitational model, the fod demand flows have been divided into 3 classes; class 1 represents the highest flows while class 3 represents the lowest ones. The subdivision into classes has been made according to the natural breaks defined by Jenks Algorithm. The natural

breaks criterion sets the limits between two classes in correspondence of discontinuities or "jumps" in the frequency distribution. We proceed with the assignment of demand flows belonging to the first class of importance (high flows); in this way it is possible to identify the paths, made up of a sequence of links at minimum cost.

To each path used by the above demand flows is given a level of importance $I_{O D}=1$; consequently, each link making up the selected routes is given importance $I_{O D}=1$.

With the same approach we proceed to assign the traffic flows of the OD matrix belonging to the second importance class, attributing an $I_{O D}=2$ importance indicator to the relative links crossed. This operation is not performed for the links that in the previous phase have already been marked with an $I_{O D}=1$ importance indicator. Finally, the same procedure is carried out for the relationships whose flows have been classified in the third importance class. Fig. 5 shows an example of an OD matrix in which the traffic flows are split into three classes. In red are identified higher flows while in green the more contained traffic flows.

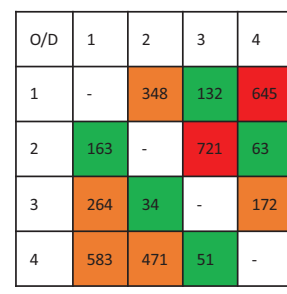

Legend
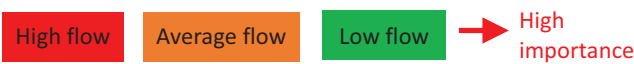

Fig. 5. Example of O/D matrix with traffic flows divided into classes. The traffic flows in the OD matrix in red give rise to the two routes characterized by a high priority of intervention. The two routes have been determined with the assignment of the $\mathrm{O} / \mathrm{D}$ matrix considering the minimum travel time.

\subsection{Link flow - Ifa}

The $I_{f a}$ indicator considers the link flow as the sum of the route flows related to the $\mathrm{O} / \mathrm{D}$ relations connecting the different zones of the territory.

The links characterized by a high flow can be considered important in terms of traffic volumes according to a punctual approach; in fact, a road link is analyzed without considering its relationships with the transport network. However, this indicator does not take into account a network approach where the aim is to ensure the practicability of the routes. Even for this indicator, the links of the road network have been divided into 3 classes according to traffic flow. Links with a high traffic value have been assigned a value of $I_{f a}=1$, while links with a low flow have 
a value of $I_{f a}=3$. A graphic representation of the outputs related to the link flow indicator is shown in Fig. 6.

Fig. 6. Representation of the outputs related to the link flow indicator $I_{f a}$ in the study area (made with GIS tools).

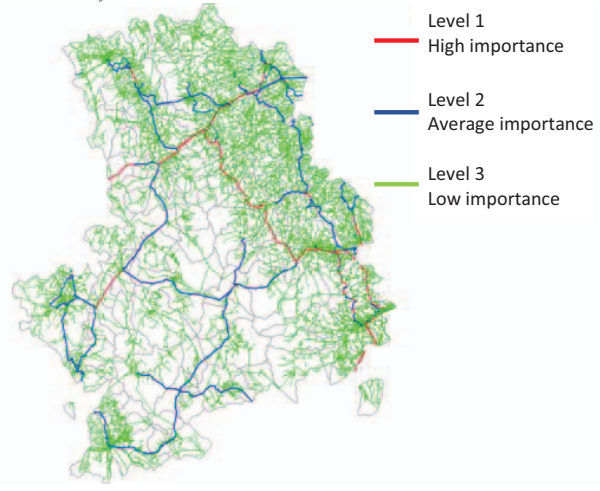

\subsection{Link flow/number of routes - Ia_np}

The indicator $I_{a n p}$ considers both the link flow and the number of paths passing over the link it. Compared to the previous indicator $\left(I_{f a}\right)$, the importance associated with a link increases if there are several routes affecting it. It means that a link is more important transport-wise if more origin/destination relations are needed. Let's proceed with the calculation of the importance indicator link flow $I_{f a}$ as explained above. Subsequently, with the same methodology, a similar $I_{N p a}$ indicator is determined based on the number of paths passing over the link $a$; also in this case a subdivision of the link into 3 classes is considered. Class 1 is characterized by the highest number of paths while class 3 identifies a situation with few paths. Fig. 7 shows an outline of the calculation of the indicator. Starting from the flows of each link and the number of paths that cross it, we can identify the links with the highest value of the indicator.

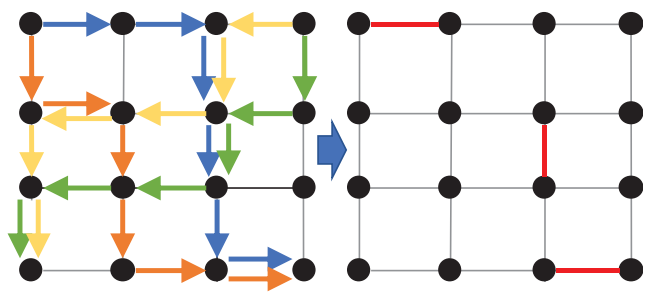

Fig. 7. Outline of the flow/number of routes indicator calculation.

The third step is to calculate the indicator $I_{a n n}$ determined by the minimum value between the two previous indicators as reported in Eq. (1):

$$
I_{a_{-} n p}=\min \left[I_{f_{a}} ; I_{N p_{a}}\right]
$$

A representation of the results for the indicator link flow importance/number of paths is shown in Fig. 8 .

Fig. 8. Representation of the outputs related to the link flow/number of routes indicator $I_{a_{-} n p}$ in the study area. (made

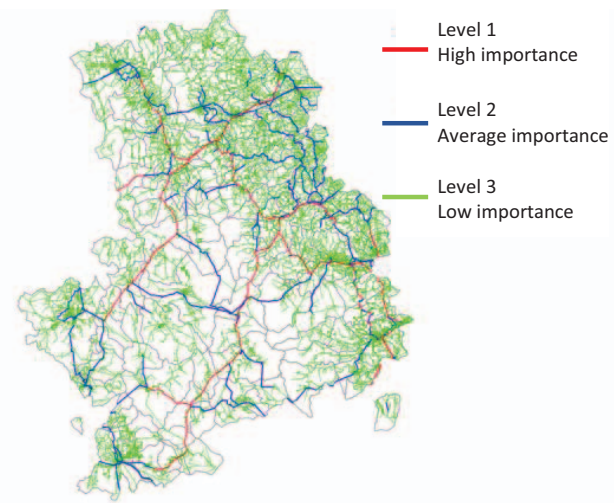

with GIS tools).

\subsection{Routes - Ipa}

This indicator combines both a punctual and a systemic analysis. The importance of a link $a$ is analyzed considering both the flow and the relationships that the link has in ensuring the continuity of the routes that cross it. With the $I_{p a}$ indicator we want to guarantee the continuity of importance on all the routes that pass through a link characterized by a link flow indicator equal to 1 or 2. The calculation procedure considers the classification of the links of the road network according to the traffic flow ( $I_{f a}$ indicator). For each link $a$ characterized by $I_{f a}=1$ (high importance) the $p_{O D}$ routes passing through it are identified. The value $I_{p a}=1$ is extended to these routes. Fig. 9 shows an outline of the indicator calculation. Starting from the two red links characterized by $I_{f a}=1$ (high importance) represented on the left, the routes that pass through the two links on the right are identified.

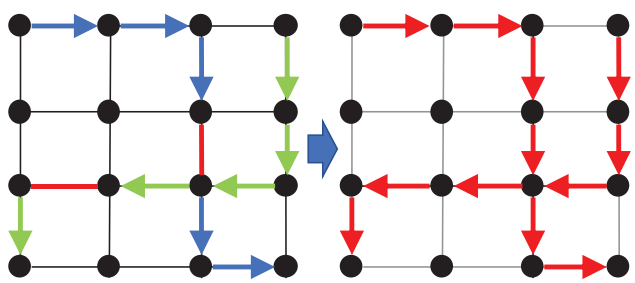

Fig. 9. Outline of the routes indicator calculation.

Fig. 10 shows the extension process of the level of importance from link to route. In this way the continuity of the routes between the different OD is guaranteed. 


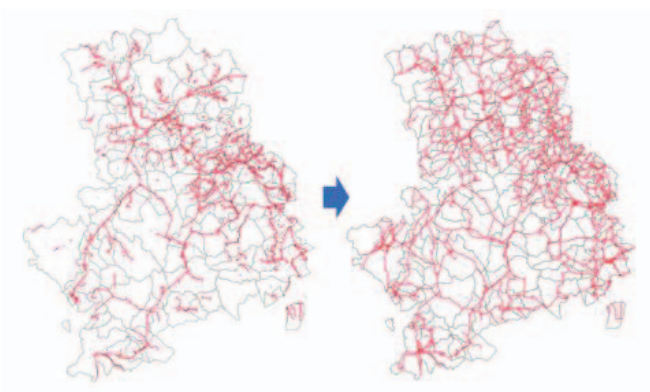

Fig. 10. Graphic representation of the process of extending the level of importance of links to all the routes that pass through them (made with GIS tools).

The procedure is repeated considering the links characterized by $I_{f a}=2$ (medium importance). The level of importance 2 of these links is extended to the routes that pass through them $\left(I_{p a}=2\right)$; this value is not extended to those paths that in the previous iteration had already been attributed a value of the indicator $I_{p a}=1$. The links characterized by link flow indicator $I_{f a}=3$ confirm their level of importance by assuming a value of the indicator $I_{p a}=2$. The output representation of the routes indicator is shown in Fig. 11.

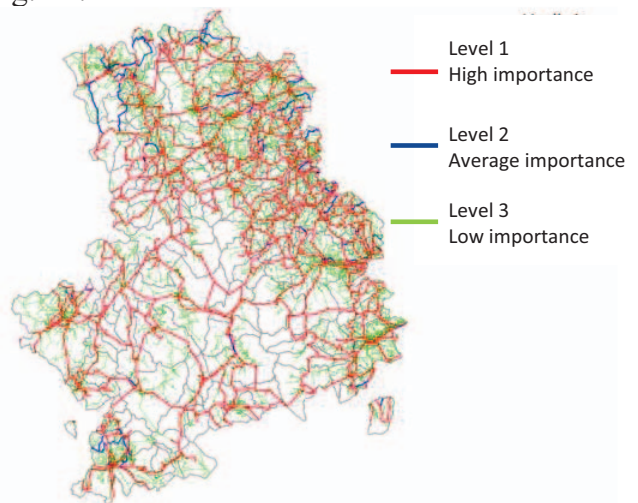

Fig. 11. Representation of the outputs related to the routes indicator $I_{p a}$ in the study area (made with GIS tools)

\subsection{Percentage route $I_{p} \%$}

The fifth indicator $I_{p} \%$ is similar to the previous one, but the criterion to define the most important links of the road network is based on the link flow/number of routes indicator $I_{a \_n p}$ and not on the link flow indicator $I_{f a}$. In addition, the extension of the importance level from the selected links to the routes does not occur for all routes passing through the selected link (as in the previous indicator), but only to routes with a higher traffic flow. The calculation procedure of the $I_{p} \%$ indicator considers the classification of the links of the road network according to the third indicator, i.e. link flow/number of routes $I_{a n}$; the three classes of importance are maintained (levels 1,2 and 3). For each link $a$, characterized by an indicator of importance $I_{a_{-} n p}=1$ (high importance), the $p_{O D}$ routes passing through it are identified and the related $f^{O D}$ route flows are considered. The paths must be ordered in decreasing order according to $f^{O D}$, as shown in the example in Table 1 for the links, 7-9 and 1-5. Note that the flow of link 7-9 is 14,632; this value is the sum of the flows of the four routes L-M, E-F, P$\mathrm{Q}$ and $\mathrm{G}-\mathrm{H}$ that cross link 7-9. The link flow 1-5 is equal to 1,569 and is determined as the sum of the two paths J-K and B-C that cross it.

Table 1. Decreasing order of $f^{O D}$ route flows for two links characterized by the indicator $I_{a_{-} n}=1$ (high importance).

\begin{tabular}{llll}
\hline $\begin{array}{l}a \\
\text { (link) }\end{array}$ & $\begin{array}{l}p_{O D} \\
\text { (route) }\end{array}$ & $\begin{array}{l}f^{O D} \\
\text { (route flow) }\end{array}$ & $\begin{array}{l}f_{a} \\
\text { (link flow) }\end{array}$ \\
\hline $7-9$ & L-M & 3,884 & \\
$7-9$ & E-F & 3,800 & 14,632 \\
$7-9$ & P-Q & 3,494 & \\
$7-9$ & G-H & 3,454 & \\
$1-5$ & J-K & 802 & 1,569 \\
$1-5$ & B-C & 767 & \\
\hline
\end{tabular}

The next step is to define a $R_{a}$ parameter that represents a percentage rate applicable to the flows of each link $a$. If we consider the link $a$ and its flow $f_{a}$ (determined by the sum of the $f^{O D}$ path flows passing through it), the $R_{a}$ rate is used to calculate a reduced link flow $f_{a-R a}$ (with $f_{a-R a}<f_{a}$ ). With reference to Table 1 , an example of calculation of the reduced link flow $f_{a-R a}$ is shown: link 7-9 is characterized by a link flow $f_{a}$ equal to 14,632 ; assuming a value of the $R_{a}$ parameter equal to $60 \%$, the reduced link flow is reported in Eq. (2):

$$
f_{a-R a}=f_{a} \cdot R_{a}=14,632 \cdot 0,6=8,779
$$

For each link, characterized by the importance indicator $I_{a n p}=1$, all paths passing through the link are identified. The extension of the importance level from link to path is done considering a subset of the routes passing through it. This subset is determined by the $R_{a}$ parameter selecting the path flows whose sum must not exceed the reduced link flow $f_{a-R a}$. For each link $a$, the procedure starts by selecting the path characterized by the highest $f^{O D}$ flow and continues adding the $f^{O D}$ flows of the following paths, until the condition reported in Eq. (3) is verified:

$$
\sum f^{O D} \leq f_{a-R a}
$$


When this condition is no longer verified, the calculation process stops and the importance level 1 of the link is extended only to the selected routes (whose sum of flows is less than $f_{a-R a}$ ). All the links that make up the selected paths are characterized by a level of importance $I_{p \%}=1$. With reference to the example in Table 1, in Table 2 it is highlighted that for link 7-9 only the first two paths (L-M and E-F) are selected in green because the sum of the two flows $(3,884$ and $3,800)$ is lower than the reduced link flow $f_{a-R a}$ $(8,779)$; if it is added also the third path P-Q (in red) in order of decreasing flow, the sum of the flows of the three paths $(3,884+3,800+3,494=$ $11,178)$ is greater than $f_{a-R a}(8,779)$ and therefore the condition in Eq. (3) would not be verified. The same reasoning is valid for the path $\mathrm{G}-\mathrm{H}$.

Table 2. Example of selection of routes to extend the importance level 1 from two links characterized by the indicator $I_{a n p}=1$. The $R a$ parameter is equal to $60 \%$.

\begin{tabular}{llll}
\hline $\begin{array}{l}a \\
\text { (link) }\end{array}$ & $\begin{array}{l}p_{O D} \\
\text { (route) }\end{array}$ & $\begin{array}{l}f^{O D} \\
\text { (route flow) }\end{array}$ & $\begin{array}{l}f_{a-R a} \\
\text { (link flow) }\end{array}$ \\
\hline $7-9$ & L-M & 3,884 & \\
$7-9$ & E-F & 3,800 & \\
$7-9$ & P-Q & 3,494 & 8,779 \\
$7-9$ & G-H & 3,454 & \\
$1-5$ & J-K & 802 & \\
$1-5$ & B-C & 767 & 941 \\
\hline
\end{tabular}

In the example in Table 2, all the links that make up the two selected paths (L-M and E-F) will be marked with a level of importance $I_{p \%}=1$. Fig. 12 shows an outline of the 4 paths that pass-through link 7-9. According to the parameter $f_{a-R a}$ only the paths L-M and E-F will have a high level of importance $\left(I_{p \%}=1\right)$.

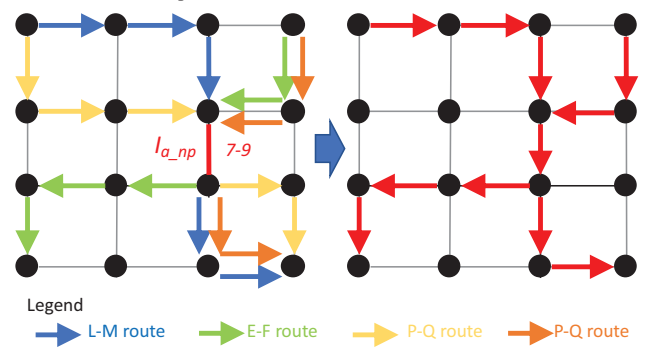

Fig. 12. Outline of the percentage routes indicator calculation.

In the case of link 1-5 only the links belonging to the J-K path (highlighted in green) will be marked with a level of importance $I_{p}=1$. In fact, in this second case, only the flow of the J-K path checks the condition in Eq. $(3)(802<941)$. We repeat the procedure considering also the links characterized by an indicator of importance $I_{a} n p$ $=2$ (medium importance). Even in this case the level of importance 2 of these links is extended to a subset of the paths passing through them, following the procedure already described for the level of importance 1 . The level of importance 2 $\left(I_{p \%}=2\right)$ cannot be extended to the links to which in the previous iteration a level of importance $I_{p \%}$ $=1$ has already been attributed. In this second iteration the $R_{a}$ parameter must be equal to the value used in the first iteration. Finally, links characterized by an initial level of importance $I_{a \_n p}=3$ (low importance), do not change classification and assume a level of importance $I_{p \%}=3$ if in previous iterations they were not affected by the extension of importance levels 1 and 2. At the end of the calculation procedure, a classification of the links in the three levels of importance $I_{p \%}$ between 1 and 3 is also obtained for this indicator, see Fig. 13, where the value 1 represents a high importance and the value 3 a low importance.

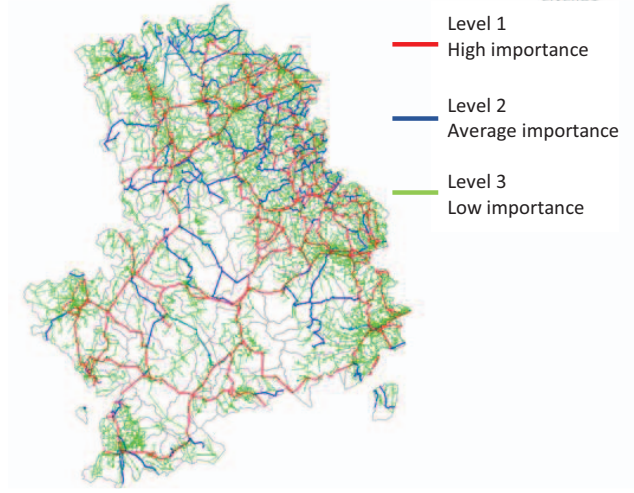

Fig. 13. Representation of the outputs related to the percentage route indicator $I_{p \%}$ in the study area (made with GIS tools).

The $R_{a}$ parameter used in the above described procedure affects the number of paths towards which the level of importance of a link $a$ is extended; $R_{a}$ can be varied according to two specific needs of the analyst. The first need concerns the case where there are resources and means to intervene only on a very limited number of links. By choosing a $R_{a}$ value of about $20-30 \%$ you can obtain a rather small subset of links of high importance, on which to concentrate the first available resources. The second need allows to intervene on a greater number of links by adopting a value of the $R_{a}$ parameter equal to about 50-60 $\%$. In this way it is possible to obtain a more numerous subsets of links characterized by an indicator of high importance.

\section{Conclusions}

The work illustrates a model developed within the framework of the agreement between Politecnico di Milano and the National Civil Protection Department; the agreement is aimed to support 
the road network recovery activities following the earthquakes that occurred in Italy between August and October 2016. The proposed model consists of two sub-models of calculation: the first concerns the development of indicators that respond to the need to guarantee the ordinary mobility of people and goods; the second considers the specific needs of Civil Protection. In this work the first sub-model has been described with reference to the development of the 5 analytical indicators to characterize the importance of each road section in relation to the road network. The Decision Support System (DSS) implemented, allows to plan and organize the operations related to road rehabilitation on the basis of objective criteria able to establish intervention priorities. The proposed model has been developed according to a flexible and modular criterion allowing, therefore, to be exported and used also in other territorial contexts. One of the most interesting aspects lies in the development of several indicators that underlie different decision-making criteria; these indicators can be used individually according to the analyst's aims. Finally, the analysis of transport demand was considered rigid and therefore not variable according to a relevant event.

\section{Acknowledgement}

The research work presented in this paper was developed within an agreement with the Italian Department of Civil Protection. The authors thank the project team for providing the data.

\section{References}

Berdica, K. (2002). An introduction to road vulnerability: What has been done, is done and should be done. Transport Policy, 117-127.

Borghetti, F., Malavasi, G. (2016a). Road accessibility model to the rail network in emergency conditions. Journal of Rail Transport Planning and Management, 237254.

Borghetti, F., Malavasi, G. (2016b). Vulnerability and accessibility of open rail routes for emergency rescue. Ingegneria Ferroviaria, 740.

Borghetti, F., Calabrese, V., Maja, R. (2017). A quantitative model for the analysis of railway accessibility with bimodal vehicle in emergency conditions. Ingegneria Ferroviaria, 72(10), 761-783

Borghetti, F., Petrenj, B., Trucco, P., Calabrese, V., Ponti, M., Marchionni, G. (in press). Multilevel Approach to Assessing the Resilience of Road Network Infrastructure. International Journal of Critical Infrastructures.
Carlson, L., Haffenden, J. A., Bassett, R., Buehring, G., Collins, W., J., Folga, M., S., Petit, F., Phillips, J.A., Verner, D.R., Whitfield, R.G. (2012). Resilience: Theory and Application. Argonne National Laboratory.

Coburn, A.W., Spence, R.J.S., Pomonis, A., 1994. Vulnerability and RiskAssessment, second ed. UNDP Disaster Management Training Programme.

Ganin, A. A., Kitsak, M., Marchese, D., Keisler, J. M., Seager, T., \& Linkov, I. (2017). Resilience and efficiency in transportation networks. Science Advances.

Ganin, A. A., Massaro, E., Gutfraind, A., Steen, N., Keisler, J. M., Kott, A., . . Linkov, I. (2016). Operational resilience: Concepts, design and analysis. Scientific Reports.

Jenelius, E., Petersen, T., \& Mattsson, L. -. (2006). Importance and exposure in road network vulnerability analysis. Transportation Research Part A: Policy and Practice, 40(7), 537-560.

Linkov, I., Fox-Lent, C., Read, L., Allen, C. R., Arnott, J. C., Bellini, E., Woods, D. (2018). Tiered approach to resilience assessment. Risk Analysis, 38(9), 1772-1780.

Ni, N., Little, R. G., Sharkey, T. C., \& Wallace, W. (2019). Modelling the recovery of critical commercial services and their interdependencies on civil infrastructures. International Journal of Critical Infrastructures, 15(4), 355-382.

Taylor, M. A. P., Sekhar, S. V. C., \& D'Este, G. M. (2006). Application of accessibility based methods for vulnerability analysis of strategic road networks. Networks and Spatial Economics, 6(3-4), 267-291.

Zhang, W., Wang, N., \& Nicholson, C. (2017). Resilience-based post-disaster recovery strategies for road-bridge networks. Structure and Infrastructure Engineering, 13(11), 1404-1413

Zio, E., Sansavini, G., Maja, R., Marchionni, G. (2008). An analytical approach to the safety of road networks. International Journal of Reliability, Quality and Safety Engineering, Vol. 15, No. 1 67-76 